\title{
FINITE $p$-GROUPS WITH A FROBENIUS GROUP OF AUTOMORPHISMS WHOSE KERNEL IS A CYCLIC $p$-GROUP
}

\author{
E. I. KHUKHRO AND N. YU. MAKARENKO
}

(Communicated by Pham Huu Tiep)

\begin{abstract}
Dedicated to Victor Danilovich Mazurov on the occasion of his 70th birthday
Abstract. Suppose that a finite $p$-group $P$ admits a Frobenius group of automorphisms $F H$ with kernel $F$ that is a cyclic $p$-group and with complement $H$. It is proved that if the fixed-point subgroup $C_{P}(H)$ of the complement is nilpotent of class $c$, then $P$ has a characteristic subgroup of index bounded in terms of $c,\left|C_{P}(F)\right|$, and $|F|$ whose nilpotency class is bounded in terms of $c$ and $|H|$ only. Examples show that the condition of $F$ being cyclic is essential. The proof is based on a Lie ring method and a theorem of the authors and P. Shumyatsky about Lie rings with a metacyclic Frobenius group of automorphisms $F H$. It is also proved that $P$ has a characteristic subgroup of $\left(\left|C_{P}(F)\right|,|F|\right)$-bounded index whose order and rank are bounded in terms of $|H|$ and the order and rank of $C_{P}(H)$, respectively, and whose exponent is bounded in terms of the exponent of $C_{P}(H)$.
\end{abstract}

\section{INTRODUCTION}

It has long been known that results on "semisimple" fixed-point-free automorphisms of nilpotent groups and Lie rings can be applied for studying "unipotent" $p$-automorphisms of finite $p$-groups. Alperin [1 was the first to use Higman's theorem on Lie rings and nilpotent groups with a fixed-point-free automorphism of prime order $p$ in the study of a finite $p$-group $P$ with an automorphism $\varphi$ of order $p$. Namely, Alperin [1 proved that the derived length of $P$ is bounded in terms of the number of fixed points $p^{m}=\left|C_{P}(\varphi)\right|$. Later the first author [9] improved the argument to obtain a subgroup of $P$ of $(p, m)$-bounded index and of $p$-bounded nilpotency class, and the second author [18] noted that this class can be bounded by $h(p)$, where $h(p)$ is Higman's function bounding the nilpotency class of a Lie ring or a nilpotent group with a fixed-point-free automorphism of order $p$. Henceforth we write for brevity, say, " $(a, b, \ldots)$-bounded" for "bounded above by some function depending only on $a, b, \ldots$ ". Further strong results on $p$-automorphisms of finite $p$-groups were obtained by Kiming [16], McKay [22, Shalev [25], Khukhro [10], Medvedev [23, 24, Jaikin-Zapirain [6], and Shalev and Zelmanov [26] giving subgroups of bounded index and of bounded derived length or nilpotency class.

Received by the editors February 14, 2013 and, in revised form, May 29, 2013.

2010 Mathematics Subject Classification. Primary 20D45; Secondary 17B40, 17B70, 20 D15.

Key words and phrases. Finite $p$-group, Frobenius group, automorphism, nilpotency class, Lie ring.

The first author was supported by the Russian Science Foundation, project no. 14-21-00065.

The second author was supported in part by the Russian Foundation for Basic Research, project no. 13-01-00505. 
The proofs of most of these unipotent results were also based on the semisimple theorems of Higman [4, Kreknin [7, and Kreknin and Kostrikin [8] on fixed-pointfree automorphisms of Lie rings.

In the present paper unipotent theorems are derived from the recent semisimple results of the authors and Shumyatsky [15, 20 about groups $G$ (and Lie rings $L$ ) admitting a Frobenius group $F H$ of automorphisms with kernel $F$ and complement $H$. The results concern the connection between the nilpotency class, order, rank, and exponent of $G$ and the corresponding parameters of $C_{G}(H)$. The more difficult of these results is about the nilpotency class, and its proof is based on the corresponding Lie ring theorem. Namely, it was proved in [15] that if the kernel $F$ is cyclic and acts on a Lie ring $L$ fixed-point-freely, $C_{L}(F)=0$, and the fixedpoint subring $C_{L}(H)$ of the complement is nilpotent of class $c$, then $L$ is nilpotent of $(c,|H|)$-bounded class (under certain assumptions on the additive group of $L$, which are satisfied in many important cases, like $L$ being an algebra over a field, or being finite). Note that examples show that the condition of $F$ being cyclic is essential. This Lie ring result also implied a similar result for a finite group $G$ with a Frobenius group $F H$ of automorphisms with cyclic fixed-point-free kernel $F$ such that $C_{G}(H)$ is nilpotent of class $c$, with reduction to nilpotent case provided by classification and representation theory arguments. The fixed-point-free action of $F$ alone was known to imply nice properties of the Lie ring (solubility of $|F|$-bounded derived length by Kreknin's theorem [7]) and of the group (solubility and well-known bounds for the Fitting height due to Thompson [27, Kurzweil [17, Turull [28], and others - although an analogue of Kreknin's theorem is still an open problem for groups). But the conclusions of the results in 15 are in a sense much stronger, due to the combination of the hypotheses on fixed points of $F$ and $H$, either of which on its own is insufficient.

We now state the unipotent version of the nilpotency class result in [15.

Theorem 1.1. Suppose that a finite p-group $P$ admits a Frobenius group $F H$ of automorphisms with cyclic kernel $F$ of order $p^{k}$. Let $c$ be the nilpotency class of the fixed-point subgroup $C_{P}(H)$ of the complement. Then $P$ has a characteristic subgroup of index bounded in terms of $c,|F|$, and $\left|C_{P}(F)\right|$ whose nilpotency class is bounded in terms of $c$ and $|H|$ only.

The proof is quite similar to the proofs of the aforementioned results of Alperin [1] and Khukhro [9], with the Lie ring theorem in [15] taking over the role of the Higman-Kreknin-Kostrikin theorem. However, first a certain combinatorial corollary of that Lie ring theorem has to be derived (Proposition 2.4). Example 3.6 shows that the condition of the kernel $F$ being cyclic in Theorem 1.1 is essential.

We now state the unipotent versions of the rank, order, and exponent results in [15]. (By the rank we mean the minimum number $r$ such that every subgroup can be generated by $r$ elements.)

Theorem 1.2. Suppose that a finite p-group $P$ admits a Frobenius group $F H$ of automorphisms with cyclic kernel $F$ of order $p^{k}$. Then $P$ has a characteristic subgroup $Q$ of index bounded in terms of $|F|$ and $\left|C_{P}(F)\right|$ such that

(a) the order of $Q$ is at most $\left|C_{P}(H)\right|^{|H|}$;

(b) the rank of $Q$ is at most $r|H|$, where $r$ is the rank of $C_{P}(H)$;

(c) the exponent of $Q$ is at most $p^{2 e}$, where $p^{e}$ is the exponent of $C_{P}(H)$. 
Note that the estimates for the order and rank are best-possible, and for the exponent close to being best-possible (and independent of $|F H|$ ). The proof is facilitated by a straightforward reduction to powerful $p$-groups. Then certain versions of the "free $H$-module arguments" are applied to abelian $F H$-invariant sections. If a finite group $G$ admits a Frobenius group of automorphisms $F H$ with complement $H$ and with kernel $F$ acting fixed-point-freely, then every elementary abelian $F H$-invariant section of $G$ is a free $k H$-module (for various prime fields $k$ ). This is exactly what provides a motivation for seeking results bounding various parameters of $G$ in terms of those of $C_{P}(H)$ and $|H|$. In the semisimple situation this fact is a basis of the results on the order and rank in [15. The exponent result in [15] is more difficult, but in our unipotent situation a simpler argument can be used based on powerful $p$-groups to produce a much better result, with the estimate for the exponent depending only on the exponent of $C_{P}(H)$.

It should be mentioned that the semisimple results on the order and rank in 15] do not assume the kernel to be cyclic. What the unipotent analogue of these results for non-cyclic kernel should be is unclear at the moment. The results of the present paper can be regarded as generalizations of the results of [15], where the kernel $F$ acts on $G$ fixed-point-freely, to the case of an "almost fixed-point-free" kernel. It is natural to expect that similar restrictions, in terms of the complement $H$ and its fixed points $C_{G}(H)$, should hold for a subgroup of index bounded in terms of $\left|C_{G}(F)\right|$ and other parameters: an almost fixed-point-free action of $F$ implying that $G$ is almost as good as when $F$ acts fixed-point-freely. In the coprime semisimple situation such restrictions were recently obtained in [13] for the order and rank of $G$, and in 14 and [19] for the nilpotency class. For the moment it is unclear how to combine these semisimple and unipotent results in a general setting, without assumptions on the orders of $G$ and $F H$; note that the results in [15] for the fixedpoint-free kernel were free of such assumptions.

\section{LIE RING TECHNIQUE}

First we recall some definitions and notation. Products in a Lie ring are called commutators. The Lie subring generated by a subset $S$ is denoted by $\langle S\rangle$ and the ideal by id $\langle S\rangle$.

Terms of the lower central series of a Lie ring $L$ are defined by induction: $\gamma_{1}(L)=$ $L ; \gamma_{i+1}(L)=\left[\gamma_{i}(L), L\right]$. By definition a Lie ring $L$ is nilpotent of class $h$ if $\gamma_{h+1}(L)=$ 0 .

A simple commutator $\left[a_{1}, a_{2}, \ldots, a_{s}\right]$ of weight (length) $s$ is by definition the commutator $\left[\ldots\left[\left[a_{1}, a_{2}\right], a_{3}\right], \ldots, a_{s}\right]$.

Let $A$ be an additively written abelian group. A Lie ring $L$ is $A$-graded if

$$
L=\bigoplus_{a \in A} L_{a} \quad \text { and } \quad\left[L_{a}, L_{b}\right] \subseteq L_{a+b}, \quad a, b \in A,
$$

where the grading components $L_{a}$ are additive subgroups of $L$. Elements of the $L_{a}$ are called homogeneous (with respect to this grading), and commutators in homogeneous elements are called homogeneous commutators. An additive subgroup $H$ of $L$ is said to be homogeneous if $H=\bigoplus_{a}\left(H \cap L_{a}\right)$; then we set $H_{a}=H \cap L_{a}$. Obviously, any subring or an ideal generated by homogeneous additive subgroups is homogeneous. A homogeneous subring and the quotient ring by a homogeneous ideal can be regarded as $A$-graded rings with the induced gradings. 
Suppose that a Lie ring $L$ admits a Frobenius group of automorphisms $F H$ with cyclic kernel $F=\langle\varphi\rangle$ of order $n$. Let $\omega$ be a primitive $n$th root of unity. We extend the ground ring by $\omega$ and denote by $\widetilde{L}$ the $\operatorname{ring} L \otimes_{\mathbb{Z}} \mathbb{Z}[\omega]$. Then $\varphi$ naturally acts on $\widetilde{L}$ and, in particular, $C_{\widetilde{L}}(\varphi)=C_{L}(\varphi) \otimes_{\mathbb{Z}} \mathbb{Z}[\omega]$.

Definition 2.1. We define $\varphi$-components $L_{k}$ for $k=0,1, \ldots, n-1$ as the "eigensubspaces"

$$
L_{k}=\left\{a \in \widetilde{L} \mid a^{\varphi}=\omega^{k} a\right\} .
$$

It is well known that $n \widetilde{L} \subseteq L_{0}+L_{1}+\cdots+L_{n-1}$ (see, for example, [5, Ch. 10]). This decomposition resembles a $(\mathbb{Z} / n \mathbb{Z})$-grading because of the inclusions $\left[L_{s}, L_{t}\right] \subseteq$ $L_{s+t(\bmod n)}$, but the sum of $\varphi$-components is not direct in general.

Definition 2.2. We refer to commutators in elements of $\varphi$-components as being $\varphi$-homogeneous.

Index Convention. Henceforth a small letter with index $i$ denotes an element of the $\varphi$-component $L_{i}$, so that the index only indicates the $\varphi$-component to which this element belongs: $x_{i} \in L_{i}$. To lighten the notation we will not use numbering indices for elements in $L_{j}$, so that different elements can be denoted by the same symbol when it only matters to which $\varphi$-component these elements belong. For example, $x_{1}$ and $x_{1}$ can be different elements of $L_{1}$, so that $\left[x_{1}, x_{1}\right]$ can be a nonzero element of $L_{2}$. These indices will be considered modulo $n$; for example, $a_{-i} \in L_{-i}=L_{n-i}$.

Note that under the Index Convention a $\varphi$-homogeneous commutator belongs to the $\varphi$-component $L_{s}$, where $s$ is the sum modulo $n$ of the indices of all the elements occurring in this commutator.

Since the kernel $F$ of the Frobenius group $F H$ is cyclic, the complement $H$ is also cyclic. Let $H=\langle h\rangle$ be of order $q$ and $\varphi^{h^{-1}}=\varphi^{r}$ for some $1 \leq r \leq n-1$. Then $r$ is a primitive $q$ th root of unity in the ring $\mathbb{Z} / n \mathbb{Z}$.

The group $H$ permutes the $\varphi$-components $L_{i}$ as follows: $L_{i}^{h}=L_{r i}$ for all $i \in$ $\mathbb{Z} / n \mathbb{Z}$. Indeed, if $x_{i} \in L_{i}$, then $\left(x_{i}^{h}\right)^{\varphi}=x_{i}^{h \varphi h^{-1} h}=\left(x_{i}^{\varphi^{r}}\right)^{h}=\omega^{i r} x_{i}^{h}$, so that $L_{i}^{h} \subseteq L_{i r}$; the reverse inclusion is obtained by applying the same argument to $h^{-1}$.

Notation. In what follows, for a given $u_{k} \in L_{k}$ we denote the element $u_{k}^{h^{i}}$ by $u_{r^{i} k}$ under the Index Convention, since $L_{k}^{h^{i}}=L_{r^{i} k}$. We denote the $H$-orbit of an element $x_{i}$ by $O\left(x_{i}\right)=\left\{x_{i}, x_{r i}, \ldots, x_{r^{q-1} i}\right\}$.

We are going to prove a combinatorial consequence of the Makarenko-KhukhroShumyatsky theorem in [15, which we state in a somewhat different form, in terms of $(\mathbb{Z} / n \mathbb{Z})$-graded Lie rings with a cyclic group of automorphisms $H$.

Theorem 2.3 ([15, Theorem 5.5 (b)]). Let $M=\bigoplus_{i=0}^{n-1} M_{i}$ be a $(\mathbb{Z} / n \mathbb{Z})$-graded Lie ring with grading components $M_{i}$ that are additive subgroups satisfying the inclusions $\left[M_{i}, M_{j}\right] \subseteq M_{i+j}(\bmod n)$. Suppose $M$ admits a finite cyclic group of automorphisms $H=\langle h\rangle$ of order $q$ such that $M_{i}^{h}=M_{\text {ri }}$ for some element $r \in \mathbb{Z} / n \mathbb{Z}$ having multiplicative order $q$. If $M_{0}=0$ and $C_{M}(H)$ is nilpotent of class $c$, then for some functions $u=u(c, q)$ and $f=f(c, q)$ depending only on $c$ and $q$, the Lie subring $n^{u} M$ is nilpotent of class $f-1$, that is, $\gamma_{f}\left(n^{u} M\right)=n^{u f} \gamma_{f}(M)=0$.

The corresponding theorems in [15] were stated about Lie rings admitting a Frobenius group $F H$ of automorphisms with cyclic kernel $F=\langle\varphi\rangle$ of order $n$. 
After extension of the ground ring, the $\varphi$-components behave like components of a $(\mathbb{Z} / n \mathbb{Z})$-grading, as we saw above. In fact, the proofs in 15 only used the grading properties of the $\varphi$-components, so that Theorem 2.3 was actually proved therein. The following proposition is a combinatorial consequence of this theorem.

Proposition 2.4. Let $f=f(c, q), u=u(c, q)$ be the functions in Theorem 2.3. Suppose that a Lie ring $L$ admits a Frobenius group of automorphisms $F H$ with cyclic kernel $F=\langle\varphi\rangle$ of order $n$ and with complement $H$ of order $q$ such that the fixed-point subring $C_{L}(H)$ of the complement is nilpotent of class $c$. Then for the $(c, q)$-bounded number $w=(u+1) f$ the $n^{w}$ th multiple $n^{w}\left[x_{i_{1}}, x_{i_{2}}, \ldots, x_{i_{f}}\right]$ of every simple $\varphi$-homogeneous commutator in $\widetilde{L}=L \otimes_{\mathbb{Z}} \mathbb{Z}[\omega]$ of weight $f$ with non-zero indices can be represented as a linear combination of $\varphi$-homogeneous commutators of the same weight $f$ in elements of the union of $H$-orbits $\bigcup_{s=1}^{f} O\left(x_{i_{s}}\right)$ each of which contains a subcommutator with zero sum of indices modulo $n$.

Remark 2.5. Similar combinatorial propositions were also proved for Lie algebras in [19] and for Lie rings whose ground ring contains the inverse of $n$ in [14].

Proof. The idea of the proof is application of Theorem 2.3 to a free Lie ring with operators $F H$. Given arbitrary (not necessarily distinct) non-zero elements $i_{1}, i_{2}, \ldots, i_{f} \in \mathbb{Z} / n \mathbb{Z}$, we consider a free Lie ring $K$ over with $q f$ free generators in the set

$$
Y=\{\underbrace{y_{i_{1}}, y_{r i_{1}}, \ldots, y_{r^{q-1} i_{1}}}_{O\left(y_{i_{1}}\right)}, \underbrace{y_{i_{2}}, y_{r i_{2}}, \ldots, y_{r^{q-1} i_{2}}}_{O\left(y_{i_{2}}\right)}, \ldots, \underbrace{y_{i_{f}}, y_{r i_{f}}, \ldots, y_{r^{q-1} i_{f}}}_{O\left(y_{i_{f}}\right)}\},
$$

where indices are formally assigned and regarded modulo $n$ and the subsets $O\left(y_{i_{s}}\right)=$ $\left\{y_{i_{s}}, y_{r i_{s}}, \ldots, y_{r^{q-1} i_{s}}\right\}$ are disjoint. Here, as in the Index Convention, we do not use numbering indices, that is, all elements $y_{r^{k} i_{j}}$ are by definition different free generators, even if indices coincide. (The Index Convention will come into force in a moment.) For every $i=0,1, \ldots, n-1$ we define the additive subgroup $K_{i}$ generated by all commutators in the generators $y_{j_{s}}$ in which the sum of indices of all entries is equal to $i$ modulo $n$. Then $K=K_{0} \oplus K_{1} \oplus \cdots \oplus K_{n-1}$. It is also obvious that $\left[K_{i}, K_{j}\right] \subseteq K_{i+j(\operatorname{modn})}$; therefore this is a $(\mathbb{Z} / n \mathbb{Z})$-grading. The Lie ring $K$ also has the natural $\mathbb{N}$-grading $K=G_{1}(Y) \oplus G_{2}(Y) \oplus \cdots$ with respect to the generating set $Y$, where $G_{i}(Y)$ is the additive subgroup generated by all commutators of weight $i$ in elements of $Y$.

We define an action of the Frobenius group $F H$ on $K$ by setting $k_{i}^{\varphi}=\omega^{i} k_{i}$ for $k_{i} \in K_{i}$ and extending this action to $K$ by linearity. An action of $H$ is defined on the generating set $Y$ as a cyclic permutation of elements in each subset $O\left(y_{i_{s}}\right)$ by the rule $\left(y_{r^{k} i_{s}}\right)^{h}=y_{r^{k+1} i_{s}}$ for $k=0, \ldots, q-2$ and $\left(y_{r^{q-1} i_{s}}\right)^{h}=y_{i_{s}}$. Then $O\left(y_{i_{s}}\right)$ becomes the $H$-orbit of the element $y_{i_{s}}$. Clearly, $H$ permutes the components $K_{i}$ by the rule $K_{i}^{h}=K_{r i}$ for all $i \in \mathbb{Z} / n \mathbb{Z}$.

Let $J={ }_{\text {id }}\left\langle K_{0}\right\rangle$ be the ideal generated by the $\varphi$-component $K_{0}$. Clearly, the ideal $J$ consists of linear combinations of commutators in elements of $Y$ each of which contains a subcommutator with zero sum of indices modulo $n$. The ideal $J$ is generated by homogeneous elements with respect to the gradings $K=\bigoplus_{i} G_{i}(Y)$ and $K=\bigoplus_{i=0}^{n-1} K_{i}$ and therefore is homogeneous with respect to both gradings. Note also that the ideal $J$ is obviously $F H$-invariant.

Let $I={ }_{\text {id }}\left\langle\gamma_{c+1}\left(C_{K}(H)\right)\right\rangle^{F}$ be the smallest $F$-invariant ideal containing the subring $\gamma_{c+1}\left(C_{K}(H)\right)$. The ideal $I$ is obviously homogeneous with respect to the 
grading $K=\bigoplus_{i} G_{i}(Y)$ and is $F H$-invariant. The fact that the ideal $I$ is $F$-invariant implies that $n I \subseteq I_{0} \oplus \cdots \oplus I_{n-1}$, where $I_{k}=I \cap K_{k}$ for $k=0,1, \ldots, n-1$. Indeed, for $z \in I$, for every $i=0, \ldots, n-1$ we have $z_{i}:=\sum_{s=0}^{n-1} \omega^{-i s} z^{\varphi^{s}} \in K_{i}$ and $n z=\sum_{i=0}^{n-1} z_{i}$. We denote $\hat{I}=I_{0} \oplus \cdots \oplus I_{n-1}$. This is an ideal of $K$, which is homogeneous with respect to both gradings $K=\bigoplus_{i} G_{i}(Y)$ and $K=\bigoplus_{i=0}^{n-1} K_{i}$. It is also $F H$-invariant, since $I$ is $F H$-invariant and the components $K_{i}$ are permuted by $F H$.

Consider the quotient Lie ring $N=K /(J+\hat{I})$. Since the ideals $J$ and $\hat{I}$ are homogeneous with respect to the gradings $K=\bigoplus_{i} G_{i}(Y)$ and $K=\bigoplus_{i=0}^{n-1} K_{i}$, the quotient ring $N$ has the corresponding induced gradings. We use indices to denote the components $N_{i}$ of the $(\mathbb{Z} / n \mathbb{Z})$-grading induced by $K=\bigoplus_{i=0}^{n-1} K_{i}$. Note that $N_{0}=0$ by the construction of $J$.

The group $H$ permutes the grading components of $N=N_{1} \oplus \cdots \oplus N_{n-1}$ with regular orbits of length $q$. Therefore elements of $C_{N}(H)$ have the form $a+a^{h}+\cdots+$ $a^{h^{q-1}}$. Hence $C_{N}(H)$ is contained in the image of $C_{K}(H)$ in $N=K /(J+\hat{I})$ and therefore $\gamma_{c+1}\left(C_{N}(H)\right)$ is contained in the image of the ideal $I$ by its construction. Then $n \gamma_{c+1}\left(C_{N}(H)\right)=0$, since $n I \subseteq \hat{I}$.

The group $H$ also permutes the $(\mathbb{Z} / n \mathbb{Z})$-grading components of $M:=n N=$ $\bigoplus_{i=0}^{n-1} M_{i}$, where $M_{i}=n N_{i}$, with regular orbits of length $q$. Therefore, $C_{M}(H)=$ $n C_{N}(H)$ and $\gamma_{c+1}\left(C_{M}(H)\right)=\gamma_{c+1}\left(n C_{N}(H)\right)=n^{c+1} \gamma_{c+1}\left(C_{N}(H)\right)=0$.

Since $N_{0}=0$, we also have $M_{0}=0$.

By Theorem 2.3 for some $(c, q)$-bounded function $u=u(c, q)$ the Lie ring $n^{u} M$ is nilpotent of $(c, q)$-bounded class $f-1=f(c, q)-1$. Consequently,

$$
n^{(u+1) f}\left[y_{i_{1}}, y_{i_{2}}, \ldots, y_{i_{f}}\right]=\left[n^{u+1} y_{i_{1}}, n^{u+1} y_{i_{2}}, \ldots, n^{u+1} y_{i_{f}}\right] \in J+\hat{I} .
$$

Note that we should take the factors $n^{u+1}$ because the elements $y_{i_{s}} \in K$ may not belong to the preimage of $M=n N$. Since both ideals $J$ and $\hat{I}$ are homogeneous with respect to the grading $K=\bigoplus_{i} G_{i}(Y)$, this means that the left-hand side is equal modulo the ideal $\hat{I}$ to a linear combination of commutators of the same weight $f$ in elements of $Y$, each of which contains a subcommutator with zero sum of indices modulo $n$.

Now suppose that $L$ is an arbitrary Lie ring satisfying the hypothesis of Proposition 2.4 , and let $\widetilde{L}=L \otimes_{\mathbb{Z}} \mathbb{Z}[\omega]$. Let $x_{i_{1}}, x_{i_{2}}, \ldots, x_{i_{f}}$ be arbitrary $\varphi$-homogeneous elements of $\widetilde{L}$. We define the homomorphism $\delta$ from the free Lie ring $K$ into $\widetilde{L}$ extending the mapping

$$
y_{r^{k} i_{s}} \rightarrow x_{i_{s}}^{h^{k}} \quad \text { for } \quad s=1, \ldots, f \quad \text { and } \quad k=0,1, \ldots, q-1 .
$$

It is easy to see that $\delta$ commutes with the action of $F H$ on $K$ and $\widetilde{L}$. Therefore $\delta\left(O\left(y_{i_{s}}\right)\right)=O\left(x_{i_{s}}\right)$ and $\delta(I)=0$, since $\gamma_{c+1}\left(C_{\widetilde{L}}(H)\right)=0$ and $\delta\left(C_{K}(H)\right) \subseteq C_{\widetilde{L}}(H)$. We now apply $\delta$ to the representation of $n^{(u+1) f}\left[y_{i_{1}}, y_{i_{2}}, \ldots, y_{i_{f}}\right]$ constructed above. Since $\delta(\hat{I}) \subseteq \delta(I)=0$, as the image we obtain a required representation of $n^{(u+1) f}\left[x_{i_{1}}, x_{i_{2}}, \ldots, x_{i_{f}}\right]$ as a linear combination of commutators of weight $f$ in elements of the set $\delta(Y)=\bigcup_{s=1}^{f} O\left(x_{i_{s}}\right)$, each of which has a subcommutator with zero sum of indices modulo $n$. 


\section{Nilpotency Class}

We begin with two lemmas that are well-known in folklore. Induced automorphisms of invariant subgroups and sections are denoted by the same letters. Fixedpoint subgroups are denoted as centralizers in the natural semidirect products.

Lemma 3.1 (see, e. g., [11, Theorem 1.6.1]). If $\alpha$ is an automorphism of a finite group $G$ and $N$ is an $\alpha$-invariant subgroup of $G$, then $\left|C_{G / N}(\alpha)\right| \leq\left|C_{G}(\alpha)\right|$.

Lemma 3.2 (see, e. g., [11, Theorem 1.6.2]). If $\alpha$ is an automorphism of a finite group $G$ and $N$ is an $\alpha$-invariant subgroup of $G$ such that $(|N|,|\alpha|)=1$, then $C_{G / N}(\alpha)=C_{G}(\alpha) N / N$.

Lemma 3.3 (see, e. g., [11, Corollary 1.7.4]). If $\varphi$ is an automorphism of order $p^{k}$ of a finite abelian p-group $A$ and $\left|C_{A}(\varphi)\right|=p^{s}$, then the rank of $A$ is at most $s p^{k}$.

The following lemma is a well-known consequence of the theory of powerful $p$ groups [21].

Lemma 3.4 (see, e. g., [12, Corollary 11.21]). If a finite p-group $P$ has rank $r$ and exponent $p^{e}$, then $|P|$ is $(p, r, e)$-bounded.

Proof of Theorem 1.1. Recall that $P$ is a finite $p$-group admitting a Frobenius group $F H$ of automorphisms with cyclic kernel $F=\langle\varphi\rangle$ of order $p^{k}$ and complement $H$ of order $q$. Let $p^{m}=\left|C_{P}(F)\right|$ and let $C_{P}(H)$ be nilpotent of class $c$. We need to find a characteristic subgroup of $(p, k, m, c)$-bounded index and of $(c, q)$-bounded nilpotency class.

Consider the associated Lie ring $L(P)=\bigoplus_{i} \gamma_{i}(P) / \gamma_{i+1}(P)$, where $\gamma_{i}$ denotes the $i$ th term of the lower central series (see, e. g., $\S 3.2$ in 11]). Extend the ground ring by a $p^{k}$ th primitive root of unity $\omega$ setting $L=L(P) \otimes_{\mathbb{Z}} \mathbb{Z}[\omega]$ and regarding $L(P)$ as $L(P) \otimes 1$. The group $F H$ naturally acts on $L$. We define the $\varphi$-components as in $\S 2$ (with $n=p^{k}$ ); recall that $p^{k} L \subseteq L_{0}+L_{1}+\cdots+L_{p^{k}-1}$. Since any $\varphi$ homogeneous commutator with zero sum of indices modulo $p^{k}$ belongs to $L_{0}$, by Proposition 2.4 we obtain

$$
p^{k(f+w)} \gamma_{f}(L)=p^{k w} \gamma_{f}\left(p^{k} L\right) \subseteq p^{k w} \gamma_{f}\left(L_{0}+L_{1}+\cdots+L_{p^{k}-1}\right) \subseteq{ }_{\mathrm{id}}\left\langle L_{0}\right\rangle
$$

for the functions $f=f(c, q), w=w(c, q)$ in that proposition. Since $L_{0}=$ $C_{L(P)}(\varphi) \otimes_{\mathbb{Z}} \mathbb{Z}[\omega]$ and $p^{m} C_{L(P)}(\varphi)=0$ by Lemma 3.1 and the Lagrange theorem, we obtain

$$
p^{k(f+w)+m} \gamma_{f}(L) \subseteq p^{m}{ }_{\text {id }}\left\langle L_{0}\right\rangle=0 .
$$

In particular, $p^{k(f+w)+m} \gamma_{f}(L(P))=0$. In terms of the group $P$ this means that the factors $\gamma_{i}(P) / \gamma_{i+1}(P)$ have exponent dividing $p^{k(f+w)+m}$ for all $i \geq f$.

By Lemmas 3.1 and 3.3, the rank of every factor $\gamma_{i}(P) / \gamma_{i+1}(P)$ is at most $m p^{k}$. Together with the bound for the exponent, this gives a bound for the order, which we state as a lemma.

Lemma 3.5. Suppose that $P$ is a finite p-group admitting a Frobenius group $F H$ of automorphisms with cyclic kernel $F=\langle\varphi\rangle$ of order $p^{k}$ and complement $H$ of order q. Let $p^{m}=\left|C_{P}(F)\right|$ and let $C_{P}(H)$ be nilpotent of class $c$. Then $\left|\gamma_{i}(P) / \gamma_{i+1}(P)\right| \leq$ $p^{(k f+k w+m) m p^{k}}$ for all $i \geq f$, where $f=f(c, q)$ and $w=w(c, q)$ are the functions in Proposition 2.4. 
Lemma 3.5 can be applied to any $F H$-invariant subgroup $Q$ of $P$. In particular, we choose $Q=\gamma_{U+1}(P\langle\varphi\rangle)$, where $U=(k f+k w+m) m p^{k}$. Clearly, $Q \leq P$, so that $\left|C_{Q}(\varphi)\right| \leq p^{m}$. By Lemma 3.5. $\left|\gamma_{i}(Q) / \gamma_{i+1}(Q)\right| \leq p^{U}$ for all $i \geq f$. On the other hand, by the well-known theorem of P. Hall [3, Theorem 2.56] we have $\left|\gamma_{i}(Q) / \gamma_{i+1}(Q)\right| \geq p^{U+1}$ if $\gamma_{i+1}(Q) \neq 1$. To avoid a contradiction we must conclude that $\gamma_{f+1}(Q)=1$. Thus, $Q$ is nilpotent of $(c, q)$-bounded class.

The automorphism $\varphi$ acts trivially on the factors of the lower central series of $P\langle\varphi\rangle$. Since $\left|C_{P\langle\varphi\rangle}(\varphi)\right|=p^{m+k}$, by Lemma 3.1 the orders of all these factors are at most $p^{m+k}$. Since the quotient $P\langle\varphi\rangle / Q$ is nilpotent of class $U$ by construction, its order is at most $p^{(m+k) U}=p^{(m+k)(k f+k w+m) m p^{k}}$, which is a $(p, k, m, c)$-bounded number. Thus, $Q$ has $(p, k, m, c)$-bounded index in $P$ and $(c, q)$-bounded nilpotency class. The subgroup $Q$ contains a characteristic subgroup $P^{p^{e}}$ for some $(p, k, m, c)$ bounded number $e$. Since the rank of $P$ is $(p, k, m, c)$-bounded, the index of $P^{p^{e}}$ in $P$ is also $(p, k, m, c)$-bounded by Lemma 3.4 .

We now produce an example showing that the condition of the kernel being cyclic in Theorem 1.1 is essential.

Example 3.6. Let $L$ be a Lie ring whose additive group is the direct sum of three copies of $\mathbb{Z}_{2}$, the group of 2 -adic integers, with generators $e_{1}, e_{2}, e_{3}$ as a $\mathbb{Z}_{2}$-module, and let the structure constants of $L$ be $\left[e_{1}, e_{2}\right]=4 e_{3}, \quad\left[e_{2}, e_{3}\right]=4 e_{1}, \quad\left[e_{3}, e_{1}\right]=4 e_{2}$. A Frobenius group $F H$ of order 12 acts on $L$ as follows: $F=\left\{1, f_{1}, f_{2}, f_{3}\right\}$, where $f_{i}\left(e_{i}\right)=e_{i}$ and $f_{i}\left(e_{j}\right)=-e_{j}$ for $i \neq j$, and $H=\langle h\rangle$ with $h\left(e_{i}\right)=e_{i+1(\bmod 3)}$. Since $L$ is a powerful Lie $\mathbb{Z}_{2}$-algebra, by [2, Theorem 9.8] the Baker-Campbell-Hausdorff formula defines the structure of a uniformly powerful pro-2-group $P$ on the same set $L$. For any positive integer $n$, the quotient of $P$ by $P^{2^{n}}=2^{n} L$ is a finite 2-group $T$. The induced action of $F H$ on $T$ is such that $\left|C_{T}(F)\right|=8$ and $C_{T}(H)$ is cyclic, while the derived length of $T$ is about $\log _{4} n$.

\section{ORDER, RANK, AND EXPONENT}

Suppose that a finite abelian group $V$ admits a Frobenius group of automorphisms $F H$ with cyclic kernel $F=\langle\varphi\rangle$ of order $n$. We can extend the ground ring by a primitive $n$th root of unity $\omega$ forming $W=V \otimes_{\mathbb{Z}} \mathbb{Z}[\omega]$ and define the natural action of the group $F H$ on $W$. As a $\mathbb{Z}$-module (abelian group), $\mathbb{Z}[\omega]=\bigoplus_{i=0}^{E(n)-1} \omega^{i} \mathbb{Z}$, where $E(n)$ is the Euler function. Hence,

$$
W=\bigoplus_{i=0}^{E(n)-1} V \otimes \omega^{i} \mathbb{Z},
$$

so that $|W|=|V|^{E(n)}$. Similarly, $C_{W}(\varphi)=\bigoplus_{i=0}^{E(n)-1} C_{V}(\varphi) \otimes \omega^{i} \mathbb{Z}$, so that $\left|C_{W}(\varphi)\right|=\left|C_{V}(\varphi)\right|^{E(n)}$.

As in $\S 2$ for $\widetilde{L}$, we define $\varphi$-components $W_{k}$ for $k=0,1, \ldots, n-1$ as the eigensubspaces

$$
W_{k}=\left\{a \in W \mid a^{\varphi}=\omega^{k} a\right\} .
$$

Recall that $W$ is an "almost direct sum" of the $W_{i}$ : namely,

$$
n W \subseteq W_{0}+W_{1}+\cdots+W_{n-1}
$$

and

(4.3) if $w_{0}+w_{1}+\cdots+w_{n-1}=0$ for $w_{i} \in W_{i}$, then $n w_{i}=0$ for all $i$. 
As in $\S 2$ we refer to elements of $\varphi$-components as being $\varphi$-homogeneous, and apply the Index Convention using lower indices of small Latin letters to only indicate the $\varphi$-component containing this element.

As before, since the kernel $F$ of the Frobenius group $F H$ is cyclic, the complement $H$ is also cyclic, $H=\langle h\rangle$, say, of order $q$, and $\varphi^{h^{-1}}=\varphi^{r}$ for some $1 \leq r \leq n-1$, which is a primitive $q$ th root of unity in $\mathbb{Z} / n \mathbb{Z}$. The group $H$ permutes the $\varphi$-components $W_{i}$ by the rule $W_{i}^{h}=W_{r i}$ for all $i \in \mathbb{Z} / n \mathbb{Z}$. For $u_{k} \in W_{k}$ we denote $u_{k}^{h^{i}}$ by $u_{r^{i} k}$ under the Index Convention.

From now on we assume in addition that $V$ is an abelian $F H$-invariant section of the $p$-group $P$ in Theorem 1.2. Recall that $|\varphi|=n=p^{k}$ and $\left|C_{P}(\varphi)\right|=p^{m}$.

Lemma 4.1. There is a characteristic subgroup $U$ of $V$ such that $|U|$ is $(p, k, m)$ bounded and

(a) $|V / U| \leq\left|C_{V}(H)\right|^{|H|}$;

(b) the rank of $V / U$ is at most $r|H|$, where $r$ is the rank of $C_{P}(H)$; and

(c) the exponent of $V / U$ is at most $p^{e}$, where $p^{e}$ is the exponent of $C_{P}(H)$.

Proof. The group $H$ acts on the set of $\varphi$-components $W_{i}$ with one single-element orbit $\left\{W_{0}\right\}$ and $\left(p^{k}-1\right) / q$ regular orbits. We choose one element in every regular $H$-orbit and let $Y=\sum_{j=1}^{\left(p^{k}-1\right) / q} W_{i_{j}}$ be the sum of these chosen $\varphi$-components. The mapping $\vartheta: y \rightarrow y+y^{h}+\cdots+y^{h^{q-1}}$ is a homomorphism of the abelian group $Y$ into $C_{W}(H)$. We claim that $p^{k} \operatorname{Ker} \vartheta=0$. Indeed, if $y \in \operatorname{Ker} \vartheta$ is written as $y=\sum_{j=1}^{\left(p^{k}-1\right) / q} y_{i_{j}}$ for $y_{i_{j}} \in W_{i_{j}}$, then $\vartheta(y)$ is equal to $y$ plus a linear combination of elements of $\varphi$-components $W_{r^{l} i_{j}}$ with all the indices $r^{l} i_{j}$ being different from the indices $i_{1}, \ldots, i_{\left(p^{k}-1\right) / q}$. Therefore the equation $\vartheta(y)=0$ implies $p^{k} y_{i_{j}}=0$ by (4.3), so that $p^{k} y=0$. Clearly, $|Y / \operatorname{Ker} \vartheta| \leq\left|C_{W}(H)\right|$, the rank of $Y / \operatorname{Ker} \vartheta$ is at most the rank of $C_{W}(H)$, and the exponent of $Y / \operatorname{Ker} \vartheta$ is at most the exponent of $C_{W}(H)$.

Let $p^{f}$ be the maximum of $p^{k}$ and the exponent of $W_{0}$, which is a $(p, k, m)$ bounded number. Then $\Omega_{f}(W) \geq W_{0}+\operatorname{Ker} \vartheta$ (where we use the standard notation $\Omega_{i}$ for the subgroup generated by all elements of order dividing $p^{i}$ ). Since

$$
p^{k} W \leq W_{0}+W_{1}+\cdots+W_{p^{k}-1}=W_{0}+Y+Y^{h}+\cdots+Y^{h^{q-1}},
$$

we obtain the following.

Lemma 4.2. The image of $p^{k} W$ in $W / \Omega_{f}(W)$ is contained in the image of $Y+$ $Y^{h}+\cdots+Y^{h^{q-1}}$ in $W / \Omega_{f}(W)$, and the image of $Y$ is a homomorphic image of $Y / \operatorname{Ker} \vartheta$.

We claim that $U=\Omega_{f+k}(V)$ is the required characteristic subgroup. The rank of the abelian group $V$ is at most $m p^{k}$ by Lemmas 3.1 and 3.3. Hence $\Omega_{f+k}(V)$ being of bounded exponent has $(p, k, m)$-bounded order. We now verify that parts (a), (b), (c) are satisfied.

(a) In the abelian $p$-group $W$ the order of the image of $p^{k} W$ in $W / \Omega_{f}(W)$ is equal to $\left|W / \Omega_{f+k}(W)\right|$. Therefore Lemma 4.2 and the fact that $|Y / \operatorname{Ker} \vartheta| \leq\left|C_{W}(H)\right|$ imply

$$
\left|W / \Omega_{f+k}\right| \leq|Y / \operatorname{Ker} \vartheta|^{|H|} \leq\left|C_{W}(H)\right|^{|H|}
$$


Clearly, $\Omega_{f+k}(W)=\Omega_{f+k}(V) \otimes_{\mathbb{Z}} \mathbb{Z}[\omega]$ and therefore $\left|\Omega_{f+k}(W)\right|=\left|\Omega_{f+k}(V)\right|^{E\left(p^{k}\right)}$. Since $|W|=|V|^{E\left(p^{k}\right)}$ and $\left|C_{W}(\varphi)\right|=\left|C_{V}(\varphi)\right|^{E\left(p^{k}\right)}$, taking the $E\left(p^{k}\right)$ th root of both sides of (4.4) gives $\left|V / \Omega_{f+k}(V)\right| \leq\left.\left|C_{V}(H)\right|\right|^{|H|}$.

(b) Similarly, the rank of the image of $p^{k} W$ in $W / \Omega_{f}(W)$ is equal to the rank of $W / \Omega_{f+k}$. By Lemma 4.2 we obtain that the rank of $W / \Omega_{f+k}(W)$ is at most $|H|$ times the rank of $Y / \operatorname{Ker} \vartheta$, which in turn is at most the rank of $C_{W}(H)$. Since the ranks are multiplied by $E\left(p^{k}\right)$ when passing from $V$ to $W$, we obtain that the rank of $V / \Omega_{f+k}(V)$ is at most $|H|$ times the rank of $C_{V}(H)$, which in turn does not exceed $r$, the rank of $C_{P}(H)$, because $C_{P}(H)$ covers $C_{V}(H)$ by Lemma 3.2 since the action of $H$ is coprime.

(c) Finally, the exponent of the image of $p^{k} W$ in $W / \Omega_{f}(W)$ is equal to the exponent of $W / \Omega_{f+k}$. By Lemma 4.2 we obtain that the exponent of $W / \Omega_{f+k}(W)$ does not exceed the exponent of $Y / \operatorname{Ker} \vartheta$ which is at most the exponent of $C_{W}(H)$ and, consequently, that of $C_{V}(H)$. Since the action of $H$ is coprime, by Lemma 3.2 the exponent of $C_{V}(H)$ (and therefore the exponent of $W / \Omega_{f+k}(W)$ as well) is at most $p^{e}$, the exponent of $C_{P}(H)$.

Proof of Theorem 1.2. Recall that $P$ is a finite $p$-group admitting a Frobenius group $F H$ of automorphisms with cyclic kernel $F$ of order $p^{k}$ with $p^{m}=\left|C_{P}(F)\right|$ fixed points of the kernel. Let $p^{s}=\left|C_{P}(H)\right|$, let $r$ be the rank of $C_{P}(H)$, and $p^{e}$ the exponent of $C_{P}(H)$. We need to find a characteristic subgroup $Q$ of $(p, k, m)$ bounded index with required bounds for the order, rank, and exponent. We can of course find such a subgroup separately for each of these parameters and then take the intersection.

By Lemmas 3.1 and 3.3 the rank of $P$ is at most $m p^{k}$. Hence $P$ has a characteristic powerful subgroup of $(p, k, m)$-bounded index by [21, Theorem 1.14]. Therefore we can assume $P$ to be powerful from the outset.

By [10] (see also [12, Theorem 12.15]), the group $P$ has a characteristic subgroup $P_{1}$ of $(p, k, m)$-bounded index that is soluble of $p^{k}$-bounded derived length at most $2 K\left(p^{k}\right)$ (where $K$ is Kreknin's function bounding the derived length of a Lie ring with a fixed-point-free automorphism of order $\left.p^{k}\right)$. Let $\mathcal{D}$ be the set of factors of the derived series of $P_{1}$. For any $V \in \mathcal{D}$, we have, by Lemma 4.1, that $|V| \leq$ $p^{g}\left|C_{V}(H)\right|^{|H|}$ for some $(p, k, m)$-bounded number $g=g(p, k, m)$. Then

$$
\left|P_{1}\right|=\prod_{V \in \mathcal{D}}|V| \leq p^{2 g K\left(p^{k}\right)} \prod_{V \in \mathcal{D}}\left|C_{V}(H)\right|^{|H|}=p^{2 g K\left(p^{k}\right)}\left|C_{P_{1}}(H)\right|^{|H|}
$$

by Lemma 3.2, since the action of $H$ is coprime. Since the rank of the powerful $p$-group $P$ is at most $m p^{k}$, by taking the $(p, k, m)$-bounded power $P_{1}^{f(p, k, m)}$ with $f(p, k, m)=p^{2 g K\left(p^{k}\right)}$ we obtain a characteristic subgroup which has $(p, k, m)$ bounded index by Lemma 3.4. The order of $P_{1}^{f(p, k, m)}$ is at most $\left|C_{P}(H)\right|^{|H|}$. Indeed, either the exponent of $P_{1}$ is at most $f(p, k, m)$ and then $P_{1}^{f(p, k, m)}=1$, or the exponent of $P_{1}$ is greater than $f(p, k, m)$ and then $\left|P_{1}: P_{1}^{f(p, k, m)}\right| \geq f(p, q, m)$, whence $\left|P_{1}^{f(p, k, m)}\right| \leq\left|C_{P_{1}}(H)\right|^{|H|} \leq\left|C_{P}(H)\right|^{|H|}$.

The powerful $p$-group $P$ has a series

$$
P>P^{p^{k_{1}}}>P^{p^{k_{2}}}>\cdots>1
$$

with uniformly powerful factors of strictly decreasing ranks. For every factor $S$ of this series having exponent, say, $p^{t}$, its subgroup $V=S^{p^{[(t+1) / 2]}}$ is abelian. 
By Lemma 4.1 the subgroup $V$ has a characteristic subgroup $U$ of $(p, k, m)$-bounded order such that the rank of $V / U$ is at most $r|H|$. The rank of $V$ is equal to the rank of $S$ and $V$ is generated by elements of order $p^{[t / 2]}$. If the rank of $S$ is higher than the rank of $U$, then there exists an element of order $p^{[t / 2]}$ that belongs to $U$ and thus $t$ should be $(p, k, m)$-bounded. Therefore the rank of $S$ can be higher than $r|H|$ only if the exponent of $S$ is $(p, k, m)$-bounded. Since the rank of $P$ is at most $m p^{k}$, all the factors in (4.5) of rank higher than $r|H|$ combine in a quotient $P / P^{p^{k} u}$ of $(p, k, m)$-bounded order; then $P^{p^{k_{u}}}$ is the required characteristic subgroup of $(p, k, m)$-bounded index and of rank at most $r|H|$.

Let $p^{v}$ be the exponent of $P$. Since in the powerful group $P$ the series $P>P^{p} \geq$ $P^{p^{2}} \geq P^{p^{3}} \geq \cdots$ is central, the subgroup $P^{p^{[(v+1) / 2]}}$ is abelian. By Lemma 4.1 the exponent of $P^{p^{[(v+1) / 2]}}$ is at most $p^{e+f}$ for some $(p, k, m)$-bounded number $f$. Hence the exponent of $P$ is at most $p^{2 e+g}$ for some $(p, k, m)$-bounded number $g=$ $g(p, k, m)$. Since the rank of $P$ is at most $m p^{k}$, by Lemma 3.4 the characteristic subgroup $P^{p^{g}}$ has $(p, k, m)$-bounded index and exponent at most $p^{2 e}$.

\section{ACKNOWLEDGMENTS}

The authors thank the referee for careful reading and helpful comments.

\section{REFERENCES}

[1] J. L. Alperin, Automorphisms of solvable groups, Proc. Amer. Math. Soc. 13 (1962), 175-180. MR0142639 (26 \#208)

[2] J. D. Dixon, M. P. F. Du Sautoy, A. Mann, D. Segal, Analytic pro-p groups, 2nd Ed., Cambridge Univ. Press, 2003.

[3] P. Hall, A Contribution to the Theory of Groups of Prime-Power Order, Proc. London Math. Soc. S2-36, no. 1, 29, DOI 10.1112/plms/s2-36.1.29. MR.1575964

[4] Graham Higman, Groups and rings having automorphisms without non-trivial fixed elements, J. London Math. Soc. 32 (1957), 321-334. MR0089204 (19,633c)

[5] Bertram Huppert and Norman Blackburn, Finite groups. II, Grundlehren der Mathematischen Wissenschaften [Fundamental Principles of Mathematical Sciences], vol. 242, SpringerVerlag, Berlin, 1982. AMD, 44. MR650245(84i:20001a)

[6] A. Jaikin-Zapirain, On almost regular automorphisms of finite p-groups, Adv. Math. 153 (2000), no. 2, 391-402, DOI 10.1006/aima.1999.1911. MR.1770935 (2001j:20027)

[7] V. A. Kreknin, Solvability of Lie algebras with a regular automorphism of finite period (Russian), Dokl. Akad. Nauk SSSR 150 (1963), 467-469. MR0157990 (28 \#1218)

[8] V. A. Kreknin and A. I. Kostrikin, Lie algebras with regular automorphisms (Russian), Dokl. Akad. Nauk SSSR 149 (1963), 249-251. MR0146230 (26 \#3752)

[9] E. I. Khukhro, Finite p-groups admitting an automorphism of order $p$ with a small number of fixed points (Russian), Mat. Zametki 38 (1985), no. 5, 652-657, 795. MR819622 (87i:20043)

[10] E. I. Khukhro, Finite p-groups that admit p-automorphisms with a small number of fixed points (Russian, with Russian summary), Mat. Sb. 184 (1993), no. 12, 53-64, DOI 10.1070/SM1995v080n02ABEH003532; English transl., Russian Acad. Sci. Sb. Math. 80 (1995), no. 2, 435-444. MR.1254804 (95d:20039)

[11] Evgenii I. Khukhro, Nilpotent groups and their automorphisms, de Gruyter Expositions in Mathematics, vol. 8, Walter de Gruyter \& Co., Berlin, 1993. MR1224233 (94g:20046)

[12] E. I. Khukhro, p-automorphisms of finite p-groups, London Mathematical Society Lecture Note Series, vol. 246, Cambridge University Press, Cambridge, 1998. MR1615819 (99d:20029)

[13] E. I. Khukhro, The rank and order of a finite group that admits a Frobenius group of automorphisms (Russian, with English and Russian summaries), Algebra Logika 52 (2013), no. 1, 99-108, 124, 127; English transl., Algebra Logic 52 (2013), no. 1, 72-78. MR3113801

[14] E. I. Khukhro and N. Yu. Makarenko, Finite groups and Lie rings with a metacyclic Frobenius group of automorphisms, J. Algebra 386 (2013), 77-104, DOI 10.1016/j.jalgebra.2013.04.008. MR 3049577 
[15] Evgeny Khukhro, Natalia Makarenko, and Pavel Shumyatsky, Frobenius groups of automorphisms and their fixed points, Forum Math. 26 (2014), no. 1, 73-112, DOI 10.1515/form.2011.152. MR 3176625

[16] Ian Kiming, Structure and derived length of finite p-groups possessing an automorphism of p-power order having exactly $p$ fixpoints, Math. Scand. 62 (1988), no. 2, 153-172. MR964222 (89i:20035)

[17] Hans Kurzweil, p-Automorphismen von auflösbaren $p^{\prime}$-Gruppen (German), Math. Z. 120 (1971), 326-354. MR0284503 (44 \#1728)

[18] N. Yu. Makarenko, Almost regular automorphisms of prime order (Russian, with Russian summary), Sibirsk. Mat. Zh. 33 (1992), no. 5, 206-208, 224, DOI 10.1007/BF00971002; English transl., Siberian Math. J. 33 (1992), no. 5, 932-934 (1993). MR1197087 (94a:20061)

[19] N. Yu. Makarenko and E. I. Khukhro, Lie algebras admitting a metacyclic Frobenius group of automorphisms, Sibirsk. Mat. Zh. 54, no. 1 (2013), 131-149; English transl., Siberian Math. J. 54 (2013), 50-64.

[20] N. Yu. Makarenko, E. I. Khukhro, and P. Shumyatskiŭ, Fixed points of Frobenius groups of automorphisms (Russian), Dokl. Akad. Nauk 437 (2011), no. 1, 20-23, DOI 10.1134/S1064562411020050; English transl., Dokl. Math. 83 (2011), no. 2, 152-154. MR2849320

[21] Alexander Lubotzky and Avinoam Mann, Powerful p-groups. I. Finite groups, J. Algebra 105 (1987), no. 2, 484-505, DOI 10.1016/0021-8693(87)90211-0. MR873681 (88f:20045)

[22] Susan McKay, On the structure of a special class of p-groups, Quart. J. Math. Oxford Ser. (2) 38 (1987), no. 152, 489-502, DOI 10.1093/qmath/38.4.489. MR916230 (88j:20019)

[23] Yuri Medvedev, p-groups, Lie p-rings and p-automorphisms, J. London Math. Soc. (2) 58 (1998), no. 1, 27-37, DOI 10.1112/S0024610798006449. MR1666066 (99m:20035)

[24] Yuri Medvedev, p-divided Lie rings and p-groups, J. London Math. Soc. (2) 59 (1999), no. 3, 787-798, DOI 10.1112/S0024610799007310. MR1709080(2001i:20042)

[25] Aner Shalev, On almost fixed point free automorphisms, J. Algebra 157 (1993), no. 1, 271282, DOI 10.1006/jabr.1993.1100. MR.1219668 (94g:20035)

[26] A. Shalev and E. I. Zel'manov, Pro-p groups of finite coclass, Math. Proc. Cambridge Philos. Soc. 111 (1992), no. 3, 417-421, DOI 10.1017/S0305004100075514. MR.1151320 (93e:20030)

[27] John G. Thompson, Automorphisms of solvable groups, J. Algebra 1 (1964), 259-267. MR0173710 (30 \#3920)

[28] Alexandre Turull, Fitting height of groups and of fixed points, J. Algebra 86 (1984), no. 2, 555-566, DOI 10.1016/0021-8693(84)90048-6. MR732266 (85i:20021)

Sobolev Institute of Mathematics, Novosibirsk, 630 090, Russia

E-mail address: khukhro@yahoo.co.uk

Sobolev Institute of Mathematics, Novosibirsk, 630 090, Russia

Current address: Université de Haute Alsace, Mulhouse, 68093, France

E-mail address: natalia_makarenko@yahoo.fr 